\title{
Integrating Retroviral Cassette Extends Gene Delivery of HSV-1 Expression Vectors to Dividing Cells
}

BioTechniques 31:394-405 (August 2001)

\author{
Pablo de Felipe, Marta \\ Izquierdo, Francisco \\ Wandosell, and Filip Lim \\ Universidad Autónoma de \\ Madrid, Madrid, Spain
}

\section{INTRODUCTION}

It has now been well recognized that the promise of gene therapy to provide effective treatments for human diseases will not be fulfilled unless sufficient investment is made into the basic science of gene transfer. Much emphasis has been placed on the development of high-efficiency delivery vectors that achieve stable gene expression. HSV-1derived vectors are highly infectious and capable of delivering genes into a wide range of both dividing and nondividing cells. However, in the case of dividing cells, because HSV vector DNA persists episomally in the nuclei and is not replicated along with the host genome, it is eventually lost from progeny cells. On the other hand, although retroviral vectors are among the most commonly used vectors in gene therapy because of their ability to stably integrate transgenes into the genomes of target cells, they offer low efficiency of gene transfer in vivo. In this paper, we describe a hybrid vector containing elements of HSV products and retroviral vectors, thus permitting the efficient delivery and retention of transgenes into cells.

In the field of gene transfer, HSV expression vectors have been particularly useful for the delivery of transgenes into postmitotic cells both in culture and in the intact organism. These vectors are comprised of a plasmid carrying minimal HSV sequences, which allows them to be packaged into virus particles with the aid of gene functions supplied by a helper virus genome (e.g., replication- or packaging-defective). The chief advantage of this vector type is that cloning manipulations are relatively easy because of the small size (5-10 kb) of the plasmid. The plasmid sequences are packaged into virus particles consisting predominantly of 150kb concatemers $(39,43,44)$. These vectors can be grown and concentrated to high titers (at least 10\%/mL) and can accommodate transgenes at least as large as $50 \mathrm{~kb}(47)$.

Retroviral vectors have long been used for stable integration of exogenous DNA sequences into target cell genomes. Most commonly used retroviral vectors lack all of the viral genes, which are instead provided in trans by packaging cells. Because of the size constraints of retroviral capsids, these vectors have a maximum size of $8-10$ $\mathrm{kb}$, with the result that the maximum free-cloning capacity is less than $8 \mathrm{~kb}$. Retroviral vectors have been widely used with small genes, such as drug resistance or color markers, but larger genes cannot be introduced in these vectors. Other limitations of retroviruses are the low titers (typically about $10^{6}$ transducing units/mL) and the existence of some cell types that cannot be infected. These include nonmitotic cells and cell types in which the proteins used as retroviral receptors are absent or blocked.

During the last few years, there have been numerous reports of the construction of chimeric viral vectors to com bine the advantages of different viruses: alphavirus-retrovirus $(19,45)$, adenovirus-adeno-associated virus (AAV) $(12,20,29,42)$, herpesvirus-AAV (18), adenovirus-retrovirus $\quad(1,3,10,11,28)$, and baculovirus-AAV (26). Interesting$1 y$, the insertion of retroviral elements into herpesviruses was first achieved simply by co-cultivation of a retrovirus 
and a herpesvirus (17), supporting the idea that retroviruses may mediate the shuffling of genetic material in and out of herpesvirus genomes during evolution (2). Savard et al. (32) constructed HSV expression vectors containing a gag-pol-env retroviral packaging transcriptional unit and demonstrated that they can be used to rescue defective lacZ retroviral vectors resident in target cell genomes. Zheng et al. (49) recently reported a hybrid vector in which a retroviral transgene cassette is delivered to target cells using a modified adenoviral vector. Sena-Esteves et al. (35) reported an elegant extension of this system in which the vector also carries a retroviral transgene. These studies show that this class of HSV expression vectors can be used as mobile launchers to fire retroviral transgenes from within a wide range of cell types in vitro or in vivo. In this paper, we have focused on the use of retroviral components to integrate product-borne transgenes into the genome of target cells.

\section{MATERIALS AND METHODS}

\section{Plasmids}

To create a plasmid backbone with an extended, multiple cloning site linker, we first constructed pHSVpuc 2 by ligating the $a m p^{R_{\text {-containing }} E c o \mathrm{RI} /}$ NdeI fragment from pHSVflag (14) to the oriS-containing EcoRI/NdeI fragment of pHSVpuc (14). A cassette containing the puromycin-resistant gene (pac) in the context of a Moloney murine leukemia virus (MMLV) retroviral vector, derived from $\mathrm{pBABEpuro}$ (24) by digestion with SalI and HindIII, was endfilled with Klenow fragment polymerase and recircularized with ligase to create pLPL. The polylinker in this plasmid was then deleted by digestion with BamHI and EcoRI, was Klenow-endfilled, and recircularized with ligase to create pLPL2.

The hybrid plasmid, pHLPL, was constructed by ligating the $P u v \mathrm{I} / S s p \mathrm{I}$ fragment of pLPL2 containing the long terminal repeat (LTR)-flanked pac gene into pHSVpuc2 linearized with $P v u \mathrm{I} / N d e \mathrm{I}$ and was Klenow-endfilled at the NdeI end. We then constructed pHGPLPL by triple ligation of pHLPL linearized by $E c o$ RI/BclI and Klenowendfilled at the $B c l$ I end, an EcoRI/SalI fragment from pHIT60 (37), and a SalI/ScaI fragment from pNCA (7). This step introduced, from pHIT60, the gag gene and a 5' fragment of the pol gene (neither the splice donor site nor the packaging signal, $\Psi$, is included in this fragment) and, from pNCA, the remaining $3^{\prime}$ region of the pol gene and a small portion of the $5^{\prime}$ env sequence.

To construct pHLP, which is identical to pHLPL except the polypurine tract (PPT) and 3' LTR have been removed, we first introduced a HindIII/EcoRI fragment containing a ClaI site and the pac gene from $\mathrm{pLPX}$ into $\mathrm{pHSV}$ puc to construct $\mathrm{pHP}$. The plasmid $\mathrm{pLPX}$ is identical to pLPX (9), except that the polylinkers cloned in the ClaI site in the two plasmids are in the opposite directions. The ClaI/Klenow-endfilled NdeI fragment of pHP containing the HSV-1 $\Psi$ was then ligated with the ClaI/ Klenow-endfilled EcoRI fragment of pHLPL containing the $5^{\prime}$ LTR and the pac gene to create pHLP. The vector, HLP, contains the polyadenylation signals present in the HSV expression vector backbone (pHSVpuc), which lies between the end of the pac gene and the HSV packaging signal "a".

Finally, to construct $\mathrm{pH} \triangle \mathrm{GPLPL}$, a deletion was introduced in pHGPLPL by digestion with EcoRI/XhoI, Klenowendfilling, and recircularization with ligase. This removed the $5^{\prime}$ portion of the gag gene, which is also present in the opposite orientation between the $5^{\prime}$ LTR element and the pac gene. The presence of this inverted repeat in pHGPLPL gives rise to spontaneous deletion mutants by homologous recombination, thus rendering the plasmid unstable during growth in bacteria. We observed no such spontaneous mutants with $\mathrm{pH} \Delta \mathrm{GPLPL}$.

\section{Southern Blots of Viral DNA}

Viral vector DNA was prepared from infected 2-2 cells using the method of Hirt (16). After digestion with selected restriction enzymes, DNA fragments were separated by agarose gel electrophoresis and transferred to nitrocellulose membranes by the method of Southern (38). A fragment of the pac gene encompassing 0.4 $\mathrm{kb}$ (from the SacII site to the stop codon) was labeled with digoxigenin (DIG) to probe the Southern blots using a DIG DNA labeling and detection kit (model 1093657; Roche Molecular Biochemicals, Barcelona, Spain).

\section{Cell Lines}

All cell lines were grown in DMEM at $37^{\circ} \mathrm{C}, 7 \% \mathrm{CO}_{2}$, and $97 \%$ relative humidity. The cell lines-VERO (African green monkey kidney epithelia), 293 (human embryonic kidney epithelia transformed by the adenovirus E1a gene), and Hs683 (human glioma) - were grown in DMEM supplemented with $10 \%$ heat-inactivated FCS, while BHK21 cells (Syrian baby ham ster kidney epithelia) were cultured in DMEM containing 5\% heat-inactivated FCS. Murine fibroblasts, NIH/3T3, were grown with $10 \%$ heat-inactivated newborn CS. 2-2 cells are derived from VERO cells and are stable transfectants containing the ICP27 gene (36).

\section{Packaging and Titering of HSV Vectors}

For complementation of the ICP27, deletion mutant $5 \mathrm{~d} 11.2$ virus (23) was grown on 2-2 cells (36). Packaging of HSV expression vectors with $5 \mathrm{dl} 1.2$ and titering of helper virus were performed as previously described $(21,22)$. Titering of infectious vector units was performed by in situ hybridization of infected cells with the same probe as used for Southern blots (a DIG-labeled pac gene fragment). Fixation, preparation, and hybridization of the cells were performed as previously described (25).

\section{Assay for Puromycin-Resistant Colony-Forming Units}

Subconfluent 100-mm plates of cells were infected with viral supernatants, trypsinized two days later, and replated at various dilutions into new $100-\mathrm{mm}$ plates with $2 \mu \mathrm{g} / \mathrm{mL}$ puromycin (3T3, 293, and Hs683) or 10 $\mu \mathrm{g} / \mathrm{mL}$ puromycin (BHK21). The cells were maintained under selection (with medium changes every 3-4 days) for two weeks $(3 \mathrm{~T} 3,293$, and BHK21) or one month (Hs683). Resistant colonies were stained with methylene blue and 
counted with the aid of a microscope to detect small colonies. To calculate the original colony-forming units (cfu) present in the viral supernatants, the final colony counts were divided by the increase in cell number during the two days before trypsinization (determined for each cell line: 3T3, 2.5; 293, 4.4; BHK21, 2.7; and Hs683, 2.3) and multiplied by the dilution factor used for replating after trypsinization. In addition to the controls shown in each experiment, all colony-forming assays included a negative control of uninfected cells. No colonies were ever detected in these controls. Improved survival of transduced cells in the colony-forming assay was achieved in later experiments (e.g., Figure 3) compared to initial tests (e.g., Figure 2) by using a lower multiplicity of infection and by replating cells after trypsinization at a higher density. All experiments were performed at least twice, and, as can be seen, conclusions about the relative effects of some of the constructs are confirmed in separate experiments using different stocks of the same viral vector or by performing the infection in different cell lines (e.g., HGPLPL produces more puromycin-resistant colonies than HLPL in the independent experiments of Figures 2 and 4).

\section{PCR Assay for Transgene Integration}

Genomic DNA was prepared from cell colonies, and PCR analysis was carried out using the following conditions: $330 \mu \mathrm{M}$ dNTPs, $0.6 \mu \mathrm{M}$ oligonucleotide primers, $2 \mathrm{mM} \mathrm{MgCl}_{2}, 10 \%$ dimethyl sulfoxide, and $1 \mu \mathrm{g}$ template DNA/50 $\mu \mathrm{L}$ reaction; denaturing pretreatment at $96^{\circ} \mathrm{C}$ for $5 \mathrm{~min}$, then $96^{\circ} \mathrm{C}$ for $1 \mathrm{~min}, 62^{\circ} \mathrm{C}$ for $30 \mathrm{~s}, 68^{\circ} \mathrm{C}$ for 3 min $30 \mathrm{~s}$, with a 2 -s extension per cycle for 32 cycles, followed by $68^{\circ} \mathrm{C}$ for 10 min. The oligonucleotides used were $\mathrm{X}$ (5'-CCCACGGTAGGTTTGGCAAGCT-3'), Y (5'-CGACGGATCCGCG GTGGCGGTCTGGACCACG-3'), and Z (5'-GGGTCTAGAGGCACCGGG CTTGCGGGTCATGCACCA-3'). To detect the integration of the transgene, the oligonucleotide pair $\mathrm{X}$ and $\mathrm{Z}$ was used, and the presence of the pac gene was confirmed using the oligonucleotide pair $\mathrm{Y}$ and $\mathrm{Z}$.

\section{RESULTS}

\section{Hybrid HSV Vectors Encoding the pac Gene in a Retroviral Integration Cassette}

To develop HSV expression vectors able to integrate their transgenes, we modified the HSV plasmid, pHSVpuc (14), by inserting the LTR elements, primer binding site, $\Psi$, and PPT of MMLV to flank a transgene (Figure 1A, pHLPL and pHGPLPL). We used the marker gene pac, which allows assay for its maintained expression by growth of transduced cells in the presence of lethal concentrations of puromycin. The transfer into the cellular genome of vector-encoded transgenes flanked by retroviral LTR elements requires $(i)$ transcription of the transgene from the $5^{\prime}$ LTR promoter, (ii) reverse transcription of the RNA followed by second-strand synthesis to yield linear dsDNA copies of the transgene, and (iii) integration of transgene copies into cellular DNA. The first step is carried out by cellular transcriptional components, while the second and third steps are mediated by retroviral reverse transcriptase and integrase functions. In MMLV, these activities result from proteolytic processing of a fusion protein derived from the gag-pol genes. Thus, to construct the vector pHGPLPL that is able to express MMLV retroviral reverse transcriptase and integrase activities, the gag-pol genes were placed under the control of the HSV-1 IE4/5 promoter, which physically overlaps the oriS sequences. In Figure 1A, IE4/5 promoter-driven transcription is to the left of oriS. Note that the plasmids are circular and displayed as linear only to facilitate comparison. As a control, we constructed $\mathrm{pHLPL}$, which contains the

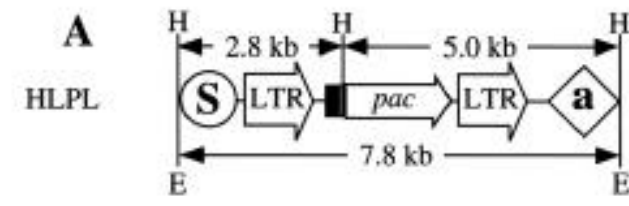

HGPLPL

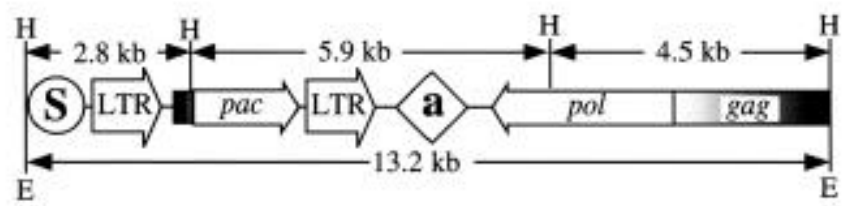

B

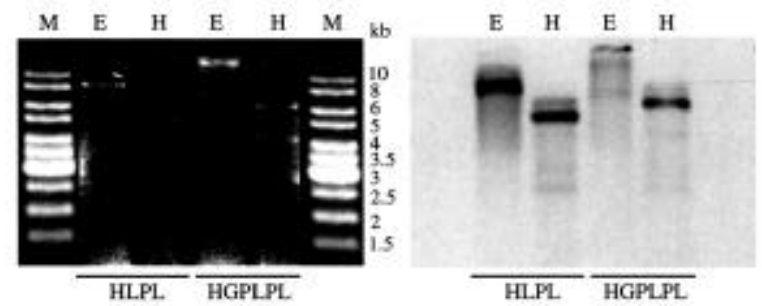

Figure 1. Characterization of hybrid HSV/retrovirus expression vectors. Two HSV expression vectors containing pac positioned between the LTR elements of MMLV were constructed. The primer binding site and $\Psi$ (see Figure 3 ) are located between the end of the $5^{\prime}$ LTR and the gag sequences (black) The vector HGPLPL also contains the MMLV gag-pol coding region. (A) Predicted restriction fragment sizes of HLPL and HGPLPL vector DNA. The elements conferring replication (S) and packaging (a) by HSV-1 are shown. H, HindIII; E, EcoRI. (B) Southern blot analysis of product DNA extracted from infected cells digested with either (E) EcoRI or (H) HindIII. In the left panel, ethidium bromide staining of the digested DNA is shown flanked by 1-kb DNA ladder size markers (M) (SM0311; MBI Fermentas, Vilnius, Lithuania). Sizes in kilobases (kb) are indicated on the right of the panel. The right panel shows the hybridization using a DNA probe containing $0.4 \mathrm{~kb}$ of the $3^{\prime}$ end of the pac gene. 
pac gene flanked by LTR elements but lacks the gag-pol genes and, thus, is unable to express retroviral reverse transcriptase or integrase activities.

The plasmids pHLPL and pHG PLPL were packaged into HSV-1 particles using the replication-defective helper virus $5 \mathrm{~d} 11.2$ as previously described $(21,22)$. Southern analysis of viral DNA using a fragment of the pac gene as a probe confirmed that the genomic structures of the two viral vectors were as predicted (Figure 1B).

\section{Retroviral Components Can Mediate Transgene Integration from HSV Vectors}

Packaged stocks of HLPL and HG PLPL were characterized by measuring the concentration of helper virus and vector (Figure 2, hlp/mL and vec/mL, respectively). Vector gene transfer was detected by in situ hybridization of infected VERO cells using a fragment of the pac gene as a probe (i.e., the same probe used in Southern analysis shown

A
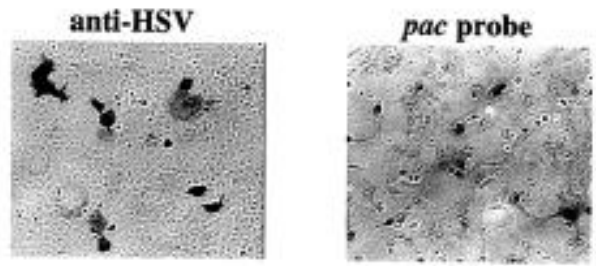

B
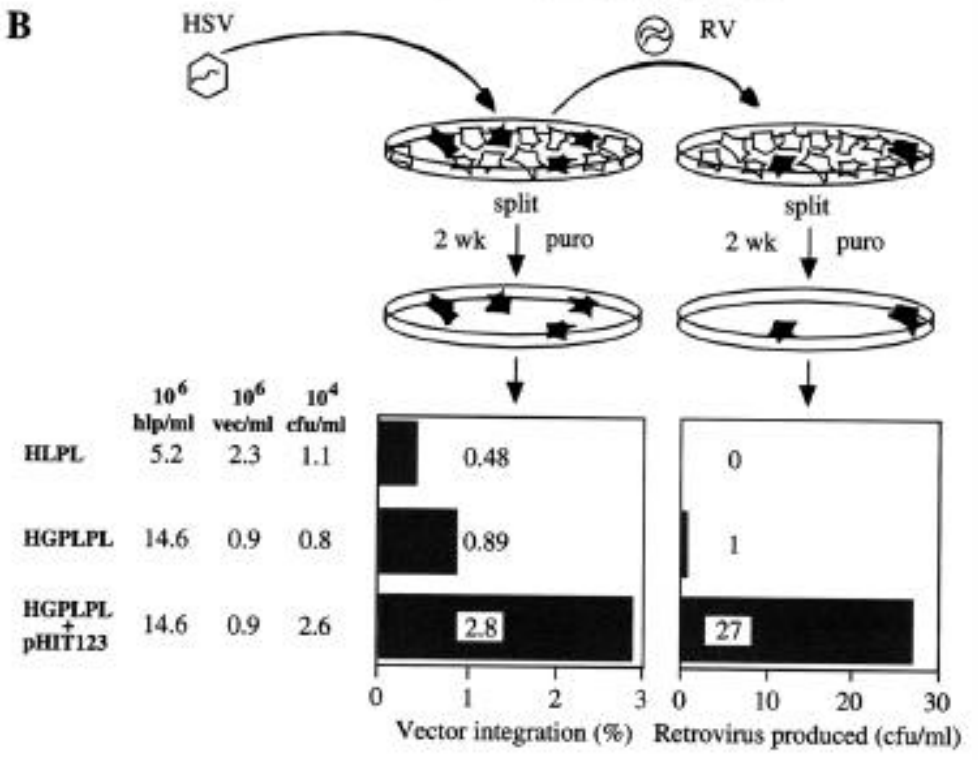

Figure 2. Long-term transgene expression of hybrid HSV/retrovirus expression vectors and testing for infectious retrovirus particles. The two HSV expression vectors, HLPL and HGPLPL, were packaged, and titers of helper virus $(\mathrm{hlp} / \mathrm{mL})$ and vector $(\mathrm{vec} / \mathrm{mL})$ were measured on 2-2 cells and VERO cells, respectively. (A) Assay for vector particles. In the example shown, VERO cells infected by HG PLPL products were fixed and stained using either anti-HSV antibodies (left) or a DIG-labeled DNA probe containing $0.4 \mathrm{~kb}$ of the $3^{\prime}$ end of the pac gene (right). Fewer cells are stained in the anti-HSV panel because a higher dilution of vector was used, compared to the staining shown in the right panel. (B) The HSV vectors (HSV) were used to infect NIH/3T3 cells, and integration of the pac gene was assayed by selection of puromycin-resistant colonies $(\mathrm{cfu} / \mathrm{mL})$. The percentage of vector particles giving rise to puromycin-resistant colonies is shown in the left bar graph (vector integration). To test for the presence of infectious retrovirus (RV) particles, supernatants from the infected NIH/3T3 cells were used to infect fresh cells, and integration of the pac gene was detected by selection of puromycin-resistant colonies (right bar graph: retroviruses produced). As a positive control, NIH/3T3 cells infected with HGPLPL were co-transfected with the plasmid pHIT123, which encodes the env gene of MMLV (HGPLPL + pHIT123), thus providing all retroviral packaging functions necessary to produce infectious retroviral particles in the supernatant. 
in Figure 1B). In contrast to the total cell staining observed using anti-HSV antibodies (Figure 2A, left panel), the staining pattern obtained using the pac gene probe was specifically nuclear (Figure 2A, right panel). The vector titer $(\mathrm{vec} / \mathrm{mL})$ was calculated by counting the number of pac-positive nuclei obtained per milliliter of viral stock. The helper virus $5 \mathrm{~d} 11.2$ was titered by plaque-forming unit assay on 2-2, the complementing cell line.

In parallel, equal volumes of the same HLPL and HGPLPL stocks were used to infect NIH/3T3 cells, and integration of the pac gene was assayed by the selection of puromycin-resistant colonies (Figure 2). We estimated the integration efficiency of each vector by the calculation of the ratio of cfu to vector units as detected by in situ hybridization (Figure 2, left bar graphs). Approximately $0.48 \%$ of the HLPL vector units gave rise to puromycin-resistant colonies, while this proportion increased to approximately $0.89 \%$ with HGPLPL, confirming that integrase activity encoded by the gag-pol genes increases the integration of the transgene.

Since both HLPL and HGPLPL contain elements of the MMLV genome, supernatants of the infected cells were tested for the presence of infectious retrovirus. After $48 \mathrm{~h}$ of infection by the HSV expression vectors, samples of the medium were used to infect fresh NIH/3T3 cells, and integration of the pac gene was assayed by selection of puromycin-resistant colonies (Figure 2, right bar graphs). No colonies were produced by infection with the supernatant from the HLPL-infected cells, and only one colony was obtained when the supernatant of the HGPLPL-infected cells was assayed. This may have arisen from remaining HGPLPL product in the culture supernatant or complementation of the defective MMLV genome by endogenous env-like genes. In contrast, complementation of HGPLPL with the MMLV env gene by transfection of the plasmid pHIT123 resulted in a significant level of infectious retrovirus in the culture medium (Figure 2, right bar graph for HGPLPL + pHIT123).

To confirm the integration of the pac gene into cellular DNA, we performed PCR analysis of genomic DNA extracted from puromycin-resistant cell colonies maintained under drug selection for one month, after infection by the HLPL and HGPLPL vectors. In our vectors, the retroviral cassettes are derived from pBABEpuro (24), which contains a small deletion in the U3 region of the $5^{\prime}$ LTR. The corresponding region of the $3^{\prime}$ LTR (Figure 3A, striped bar) is duplicated to the $5^{\prime}$ LTR during reverse transcription and integration (Figure 3A, arrow), thus providing a means to distinguish between the viral vector and the integrated provirus. Figure $3 \mathrm{~B}$ shows that, by using primers $\mathrm{X}$ and $\mathrm{Z}$, a $2.1-\mathrm{kb}$ band corresponding to the region between the $5^{\prime}$ end of the $5^{\prime}$

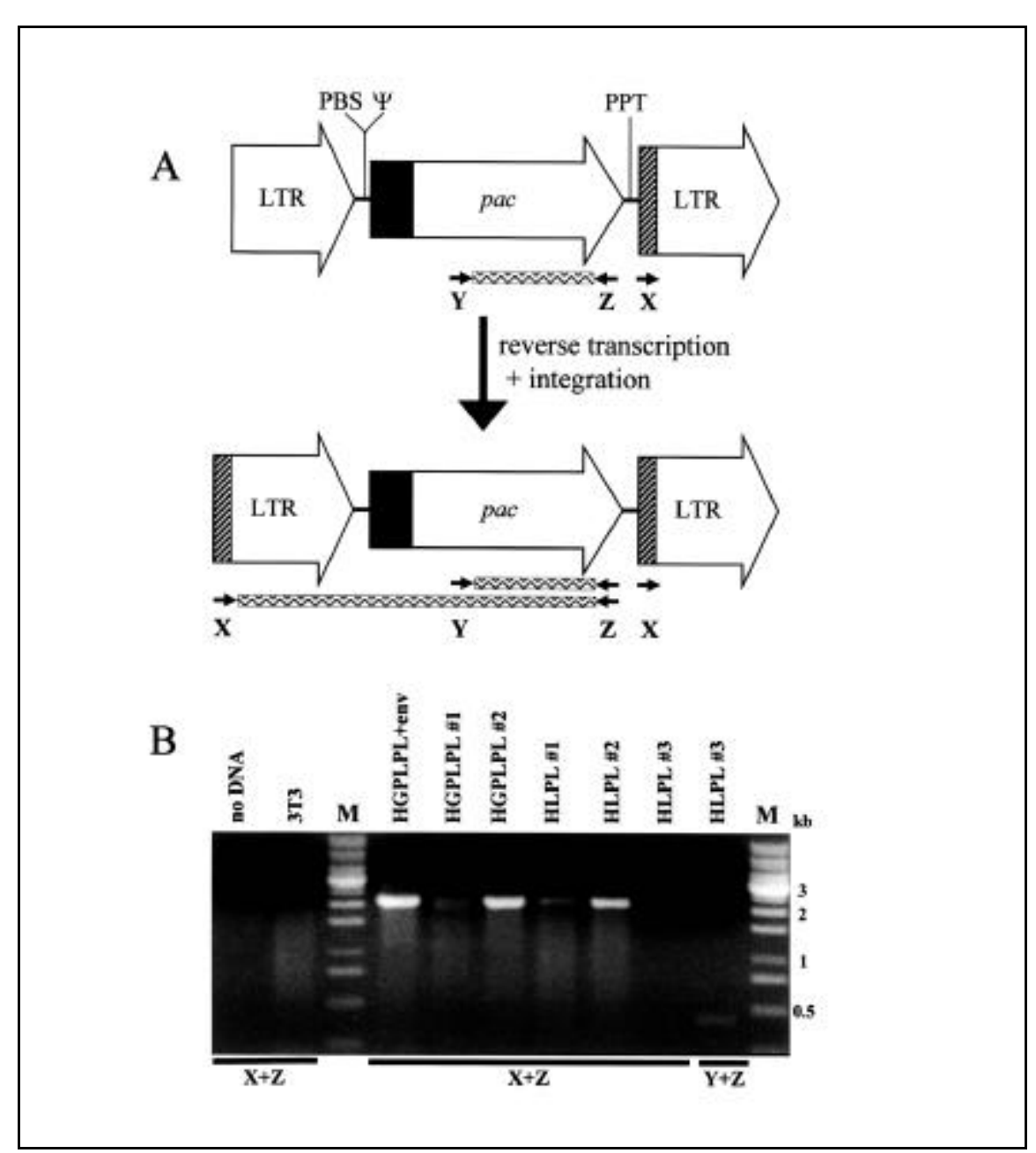

Figure 3. PCR analysis of transgene integration in NIH/3T3 cells infected by hybrid HSV/retrovirus expression vectors. (A) Schematic showing the structure of the integrating retroviral cassette containing pac positioned between the LTR elements of MMLV. The primer binding site and $\Psi$ are located between the end of the $5^{\prime}$ LTR and the gag sequences (black), while the PPT is located between the pac gene and the $3^{\prime}$ LTR. The $3^{\prime}$ LTR contains a short region of the U3 region that is deleted in the $5^{\prime}$ LTR (striped box) to which the primer $\mathrm{X}$ binds. Primer $\mathrm{Y}$ binds near the $5^{\prime}$ end of the pac gene, and primer $\mathrm{Z}$ binds at the $3^{\prime}$ end of the pac gene, thus enabling amplification of a 0.4-kb PCR product. Reverse transcription and integration of the transgene result in repair of the deleted U3 region in the $5^{\prime}$ LTR, thus enabling PCR amplification of a product by the primer pair X and Z. (B) Gel electrophoresis of PCR products from genomic DNA extracted from puromycin-resistant clonal populations derived from NIH/3T3 cells infected by HSV/retrovirus vectors. Using the primers X and Z, a 2.1-kb band could be amplified from four independent clones transduced either by HGPLPL (HGPLPL, nos. 1 and 2) or HLPL (HLPL, nos. 1 and 2). This band was not detected in PCR performed on clone HLPL no. 3, presumably because reverse transcription had not occurred. However, the presence of the pac gene could be demonstrated in these cells, as observed by the $0.4-\mathrm{kb}$ band amplified using primers $\mathrm{Y}$ and $\mathrm{Z}$. As negative controls, PCR was performed in the absence of DNA or using DNA from uninfected NIH/3T3 cells. As a positive control, PCR was performed on DNA from cells transduced by infectious retrovirus generated by complementation of HGPLPL with the env gene (see Figure 2B). The sizes in kilobases (kb) of some of the markers (M) are indicated on the right. 
LTR and the $3^{\prime}$ end of the pac gene can be amplified from DNA of four independent clonal populations infected by the chimeric vectors (Figure 3B, HG PLPL, nos. 1 and 2, and HLPL, nos. 1 and 2) or positive control cells transduced by infectious retrovirus made by complementation of HGPLPL with the env gene in pHIT123 (Figure 3B, HG PLPL + env). This PCR product was not observed when the amplification was performed in the absence of DNA (Figure 3B, no DNA) or on DNA extracted from uninfected NIH/3T3 cells. In one puromycin-resistant clone (Figure 3B, HLPL no. 3) the 2.1-kb band was not detected, but PCR amplification of a $0.4-\mathrm{kb}$ band using primers $\mathrm{Y}$ and $\mathrm{Z}$ confirmed the presence of the pac gene (Figure 3B, penultimate lane). In this clonal population, integration of the pac gene probably occurred indepen-

A

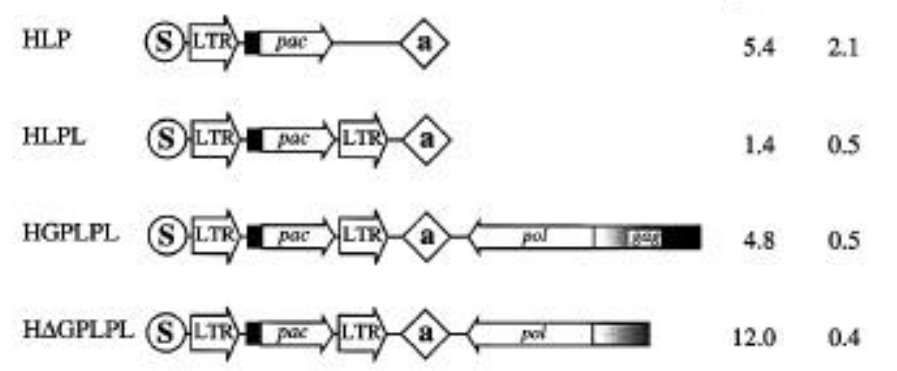

B

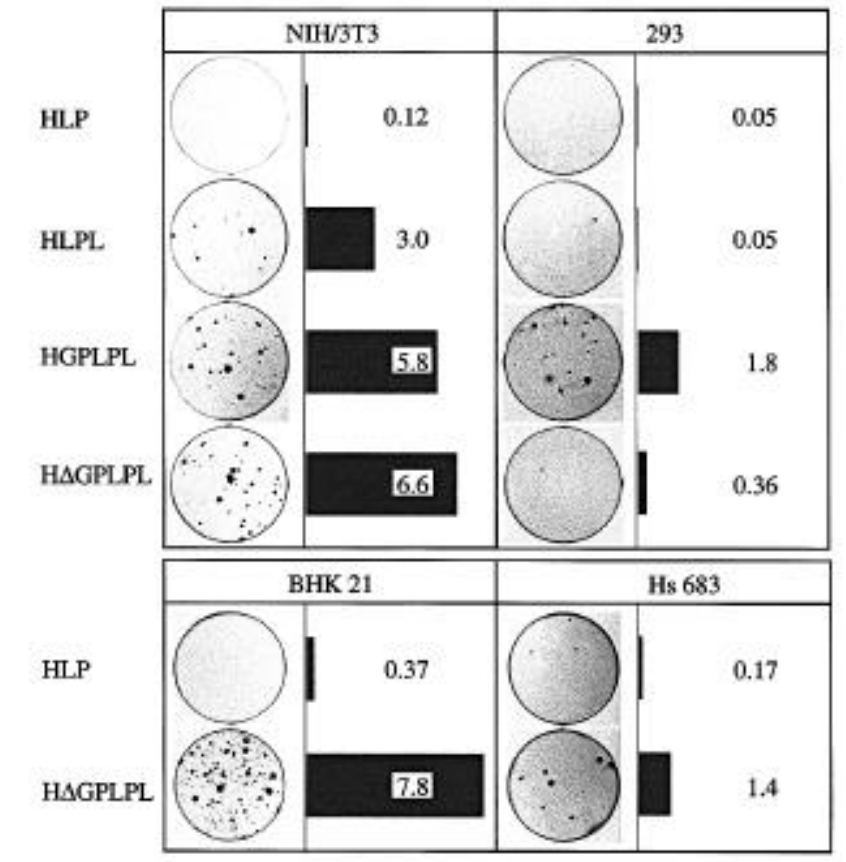

Figure 4. Role of the retroviral elements in long-term transgene expression of hybrid HSV/retrovirus expression vectors in murine and human cells. (A) Structure of four hybrid HSV/retrovirus expression vectors - HLP, HLPL, HGPLPL and HGAPLPL-differing in content of retroviral components (LTR, gag, and $\mathrm{pol}$ ). The helper $(\mathrm{hlp} / \mathrm{mL})$ and vector $(\mathrm{vec} / \mathrm{mL})$ titers of the stocks used in this experiment were measured on 2-2 cells and NIH/3T3 cells, respectively. (B) One thousand vector units of each viral stock were used to infect NIH/3T3 murine fibroblasts or 293 human kidney epithelia, and integration of the $p a c$ gene was detected by selection of puromycin-resistant colonies (photo insets). The number of cfu/vector unit obtained in each case is shown in the bar graphs. (C) BHK21 hamster kidney epithelia or Hs683 human glioma were infected with 1000 vector units HLP or HAGPLPL, and integration of the pac gene was detected by selection of puromycin-resistant colonies (photo insets). The number of cfu/vector unit obtained in each case is shown in the bar graphs. 
dently of retroviral elements, caused by mere transfection of vector DNA, and, thus, the $\mathrm{X}$ primer binding site was not regenerated at the $5^{\prime} \mathrm{LTR}$.

\section{$3^{\prime}$ LTR Is Necessary for Integration of the Retroviral Transgene Cassette}

Figure 2 shows that the level of integration we observed with our control vector, HLPL, is surprisingly high considering the negligible background levels that we (data not shown) and others $(18,30)$ have observed with other herpesvirus vectors containing only $\mathrm{HSV}-1$ elements. To ascertain whether endogenous cellular activities were mediating the integration of HLPL transgene cassette, we constructed the hybrid vector HLP (Figure 4A, top block diagram) in which the PPT and 3' LTR downstream of the pac gene are missing and, thus, preclude the action of retroviral reverse transcriptase and integrase functions. Polyadenylation signals present in the pHSVpuc lie between the end of the pac gene and the HSV packaging signal "a", thus ensuring correct termination of the pac gene mRNA.

A fourth viral vector was constructed to eliminate the problem of recom binational instability observed with the plasmid pHGPLPL. Because of the presence of an inverted repeat (Figure 4A, shaded boxes, shows that sequences from the $5^{\prime}$ portion of the gag gene are also present in the opposite orientation at the $5^{\prime}$ end of pac), growth of the plasmid pHGPLPL in bacteria gives rise to spontaneously deleted recombinants at high frequency. The MMLV gag and pol genes share a com mon open reading frame that gives rise to a Gag-pol fusion polyprotein. This is processed by a protease encoded in the $3^{\prime}$ portion of gag to give rise to the mature retroviral reverse transcriptase and integrase proteins, which are derived from the $5^{\prime}$ and $3^{\prime}$ portions of pol, respectively. Thus, the $5^{\prime}$ portion of the gag gene, which is necessary in intact retroviruses to generate the structural proteins for virion maturation, is dispensable in our system. We therefore constructed pH $\triangle$ GPLPL (Figure 4A, fourth block diagram) by deleting the $5^{\prime}$ portion of the gag gene. This plasmid is stable to passage in bacteria and does not give rise to spontaneous rearrangements.

The four plasmids-pHLP, pHLPL, pHGPLPL, and pH $\triangle$ GPLPL-were packaged into HSV particles using the replication-defective helper, 5d11.2, and helper and vector titers determined (Figure $4 \mathrm{~A}, \mathrm{hlp} / \mathrm{mL}$ and $\mathrm{vec} / \mathrm{mL}$ ) as for the experiment shown in Figures 1 and 2. We then determined the transgene integration capacity of each vector by using 1000 vector units to infect NIH/3T3 mouse fibroblasts or 293 human kidney epithelia (Figure 4B). As in the previous experiment, puromycin-resistant colonies obtained after two weeks were counted, and the percentage of original expression vector units giving rise to resistant colonies was calculated as a measure of integration efficiency. As previously observed, the number of resistant NIH/3T3 colonies obtained using HG PLPL was approximately double that obtained using HLPL. However, our new negative control HLP gave rise to only $2 \%$ of the number of colonies obtained using HGPLPL, indicating at least a 50-fold increase in the frequency of transgene integration using our system. The absolute numbers of $\mathrm{cfu}$ obtained in this experiment were higher than those observed in the experiment shown Figure 2, probably because of changes introduced into the assay to optimize the survival of the transduced cells during the drug selection process (see Materials and Methods). Significantly, in the human cell line 293, while a large number of puromycin-resistant colonies was obtained using HGPLPL, the number obtained using HLPL was very low and similar to that obtained using the negative control HLP. Our interpretation of this effect is that endogenous activities, present in the murine cells but not in the human cells, are able to at least partially substitute for retroviral reverse transcriptase and integrase, mediating the integration of the LTRflanked transgene cassette into the host genome. Curiously, the integration efficiency of $H \triangle$ GPLPL is similar to that of HGPLPL in NIH/3T3 cells but lower in 293 cells. It is possible that our particular deletion of the $5^{\prime}$ portion of gag results in decreased processing of the $\Delta$ Gag-pol fusion protein. In human cells, this reduction is reflected in lower integration efficiency; whereas, in murine cells, complementation by endogenous activities may restore levels of processing to those of HGPLPL.

\section{Various Cell Types Yield \\ Puromycin-Resistant Colonies when Infected with Hybrid HSV Vectors}

The above experiments clearly show the advantages of hybrid HSV/retroviral expression vectors over conventional HSV products for gene delivery into dividing cells. Next, we examined the ability of these vectors to solve the difficulties experienced with conventional retrovirus vectors. As a model system, we chose BHK21, a hamster kidney epithelial cell line known to be resistant to retroviral infection because of $\mathrm{N}$-linked glycosylation of membrane surface proteins in these cells [this blockage to infection can be reverted with tunicamycin (48)]. As shown in Figure 4C, left panel, when 1000 vector units of HAPLPL were used to infect BHK21 cells, a transgene integration efficiency $(7.8 \%)$ similar to that obtained in NIH/3T3 was obtained, while infection of the hamster cells with the HLP product yielded negligible puromycin-resistant cells (Figure $4 \mathrm{C}$, top of left panel). As a comparison, we also infected BHK21 cells in parallel with the pac-transducing retroviral vector LPX (9), produced with the ecotropic packaging cell line $\Psi \mathrm{CRE}$, and using quantities with previously determined titers of 1000 and $10000 \mathrm{cfu}$ in NIH/3T3 cells. No puromycin-resistant colonies were observed, demonstrating that hybrid HSV/retroviral vectors do indeed extend target range to cell types not infectable by retroviruses. It should be noted that our procedure for measuring the relative integration efficiencies of the various vectors probably results in an understatement of the absolute efficiency of stable transduction in some of the cell lines tested. This is because different cell types display different susceptibilities to infection by HSV-1 products, whereas our virus titers were measured only in VERO and NIH/3T3 cells, in which similar values were obtained. Another factor that may lower the absolute number of puromycin-resistant colonies in our assays is the presence of helper virus. The $5 \mathrm{~d} 11.2$ mutant, in spite of being replication incompetent, provokes cytopathic effects in infected cells, thus 


\section{Research Report}

raising their susceptibility to puromycin. Nevertheless, it is clear that the hybrid vectors do mediate transgene integration because they gave rise to more puromycin-resistant colonies in spite of being mixed with a higher proportion of helper virus than the negative controls (Figures 2 and 4). Packaging of these vectors using helper-free systems $(31,41)$ would reduce the interference of the viral delivery system in drug selection assays, but, to date, the vector stocks produced in our hands by helperfree packaging have been too dilute to use for these experiments.

In examining the target cell range of our hybrid HSV/retroviral vectors, another cell line of interest for us was Hs683, which we have previously used as a human glioma model to show how retroviral vectors expressing a viral thymidine kinase can be used for gene therapy (9). Because of the slow growth rate of these cells, it was necessary to culture them in selective medium (containing $2 \mu \mathrm{g} / \mathrm{mL}$ puromycin) for one month to obtain visible colonies, after which a much higher transgene integration efficiency was apparent for the vector HAGPLL compared to the negative control HLP (Figure 4C, right box), as obtained for all other cell types tested. These results indicate the potential of these vectors to genetically modify dividing cells for gene therapy.

\section{DISCUSSION}

In first-generation HSV expression vectors, maximum transgene expression is transient and reaches peak levels between two and four days after gene transfer $(21,40)$. After this expression, levels diminish and become virtually undetectable after one month, even though vector DNA persists in the infected cells. The HSV-1 LAT2 promot- er has been shown to be active in the absence of HSV-1 lytic gene expression (5), and expression vectors incorporating cellular promoters have also im proved the stability of transgene expression (8). However, these effects may be cell-type specific, particularly in the case of the LAT2 promoter. The use of these vectors is, in any case, limited to nondividing or slow-growing cell populations because vector DNA is not replicated and, thus, is eventually lost by dilution after several cell divisions.

Several hybrid vectors have been developed to achieve transgene retention in dividing cells. Chimeric products that incorporate elements of EpsteinBarr virus (EBV) allow autonomous episomal replication of the vector in primate cells (46), while those that incorporate AAV elements mediate genomic integration of the transgene in human cells (18). The hybrid vectors described here offer the advantage of 
retroviral vectors to genetically convert dividing cells of other species in vitro and in vivo. This is combined with the high infectivity and wide target cell range of HSV products and the potential to deliver much larger DNA fragments than retroviral vectors. Using the test transgene $p a c$, our results indicate that up to $8 \%$ of hybrid vector-infected target cells can give rise to puromycinresistant colonies. Significantly, we have maintained such transduced cells under drug selection for as long as one month, indicating that not only is transgene DNA retained in these cells but also that expression is stable over this period. It is most likely that the major contributions to this effect are the replicative amplification of the transgene by the retroviral pol gene function and the constitutive transcriptional activity of the MMLV LTR. A similar mechanism has been proposed to account for the extended expression observed using chimeric HSV/AAV expression vectors $(13,18)$.

Hybrid HSV/retroviral vectors offer an extended target cell range; they are retained in dividing cell populations (unlike vectors containing only HSV com ponents) and can deliver genes to cells not susceptible to retroviral infection, such as those lacking functional retroviral receptors or postmitotic and differentiated cells. Thus, these vectors are useful for gene transfer applications in a wide range of cell lines and tissues, such as neurons, glia, fibroblasts, and hematopoietic cells. Sena-Esteves et al. (35) reported the construction of similar hybrid vectors containing retroviral com ponents, with the objective of converting target cells into retrovirus producer cells. The analysis of the supernatants of infected cells showed encouraging levels of retrovirus that was able to further deliver the transgene to surrounding cells.

One consideration for the use of chimeric viral vectors is safety. Murine cells contain a large number of MMLVlike genomes $(4,6,15,27,33,34)$, some of which could potentially complement the gag-pol genes in our vectors to produce infectious retrovirus. In our experiments, we did not detect infectious retroviruses carrying the pac transgene, which would arise from complementing endogenous env genes. However, in murine cells, we observed a relatively high level of integration of a control vector containing the LTR-flanked pac transgene but lacking the gag-pol genes. This was apparently due to endogenous Gag- and Pol-like activities, and such complementation was not observed in human cells.

Our results indicate that the retroviral reverse transcriptase and integrase functions of MMLV can be successfully used in the context of HSV expression vectors to direct integration of a retroviral transgene cassette into the target cell genome. During construction of these hybrid vectors, we experienced some difficulties with instability of some of the plasmids because of their large size and the presence of repeated sequences. A deletion mutant was made to solve this problem, but integration efficiency was somewhat compromised in certain cell types with this vector. This suggests that it may be appropriate to further minimize vector size and optimize the functions necessary for transgene integration so that these vectors can fulfill their potential for gene delivery applications.

\section{ACKNOWLEDGMENTS}

We gratefully acknowledge A.J. Kingsman for plasmids pHIT 60 and pHIT 123 and 293 cells, Antonio Talavera for plasmid pNCA, Esteban Domingo for BHK21 cells, Priscilla Schaffer for the $5 \mathrm{~d} 11.2$ mutant HSV-1 virus, and Rozanne Sandri-Goldin for the 2-2 cells. We thank Marta Vaz for expert technical assistance and Jesus Avila for his generosity in making laboratory resources available for this work.

The Centro de Biologia Molecular is the recipient of an institutional grant from the Fundacion Ramon Areces. P.F. was supported by a Research Personnel Training grant from the Comunidad Autonoma de Madrid, and F.L. was supported by a biotechnology grant from the Spanish Ministry of Education and Culture.

\section{REFERENCES}

1.Bilbao, G., M. Feng, C. Rancourt, W. Jackson, Jr., and D.T. Curiel. 1997. Adenoviral/retroviral vector chimeras: a novel strategy to achieve high-efficiency stable transduction in vivo. FASEB J. 11:624-634.
2.Brunovskis, P. and H.-J. Kung. 1996. Retrotransposition and herpesvirus evolution. Virus Genes 1 1:259-270.

3.Caplen, N.J., J.N. Higginbotham, J.R. Scheel, N. Vahanian, Y. Yoshida, H. Hamada, R.M. Blaese, and W.J. Ramsey. 1999. Adeno-retroviral chimeric viruses as in vivo transducing agents. Gene Ther. 6:454-459.

4.Chakraborty, A.K., M.A. Zink, and C.P. Hodgson. 1994. Transmission of endogenous VL30 retrotransposons by helper cells used in gene therapy. Cancer Gene Ther. 1:113-118.

5.Chen, X., M.C. Schmidt, W.F. Goins, and J.C. Glorioso. 1995. Two herpes simplex virus type 1 latency-active promoters differ in their contributions to latency-associated transcript expression during lytic and latent infections. J. Virol. 69:7899-7908.

6.Colicelli, J. and S.P. Goff. 1987. Identification of endogenous retroviral sequences as potential donors for recombinational repair of mutant retroviruses: positions of crossover points. Virology 160:518-522.

7.Colicelli, J. and S.P. Goff. 1988. Sequence and spacing requirements of a retrovirus integration site. J. Mol. Biol. 199:47-59.

8.Costantini, L.C., J.C. Bakowska, X.O. Breakefield, and O. Isacson. 2000. Gene therapy in the CNS. Gene Ther. 7:93-109.

9.de Felipe, P., V. Martin, M.L. Cortes, M. Ryan, and M. Izquierdo. 1999. Use of the 2A sequence from foot-and-mouth disease virus in the generation of retroviral vectors for gene therapy. Gene Ther. 6:198-208.

10.Duisit, G., A. Salvetti, P. Moullier, and F.L. Cosset. 1999. Functional characterization of adenoviral/retroviral chimeric vectors and their use for efficient screening of retroviral producer cell lines. Hum. Gene Ther. 10:189-200.

11.Feng, M., W. Jackson, Jr., C.K. Goldman, C. Rancourt, M. Wang, S.K. Dusing, G. Siegal, and D.T. Curiel. 1997. Stable in vivo gene transduction via a novel adenoviral/retroviral chimeric vector. Nat. Biotechnol. 15:866-870.

12.Fisher, K.J., W.M. Kelley, J.F. Burda, and J.M. Wilson. 1996. A novel adenovirus-adenoassociated virus hybrid vector that displays efficient rescue and delivery of the AAV genome. Hum. Gene Ther. 7:2079-2087.

13.Fraefel, C., X.O. Breakefield, and D.R. Jacoby. 1998. HSV-1 amplicon, p. 63-82. In E.A. Chiocca and X.O. Breakefield (Eds.), Gene Therapy for Neurological Disorders and Brain Tumours. Humana Press, Totowa, NJ.

14.Geller, A.I., M.J. During, J.W. Haycock, A. Freese, and R. Neve. 1993. Long-term increases in neurotransmitter release from neuronal cells expressing a constitutively active adenylate cyclase from a herpes simplex virus type 1 vector. Proc. Natl. Acad. Sci. USA 90:7603-7607.

15.Hatzoglou, M., C.P. Hodgson, F. Mularo, and R.W. Hanson. 1990. Efficient packaging of a specific VL30 retroelement by psi 2 cells which produce MoMLV recombinant retroviruses. Hum. Gene Ther. 1:385-397.

16.Hirt, B. 1967. Selective extraction of polyoma DNA from infected mouse cell cultures. J. Mol. Biol. 26:365-369.

17.Isfort, R., D. Jones, R. Kost, R. Witter, and H.-J. Kung. 1992. Retrovirus insertion into herpesvirus in vitro and in vivo. Proc. Natl. 
Acad. Sci. USA 89:991-995.

18.Johnston, K.M., D. Jacoby, P.A. Pechan, C. Fraefel, P. Borghesani, D. Schuback, R.J. Dunn, F.I. Smith et al. 1997. HSV/AAV hybrid amplicon vectors extend transgene expression in human glioma cells. Hum. Gene Ther. 8:359-370.

19.Li, K.J. and H. Garoff. 1996. Production of infectious recombinant Moloney murine leukemia virus particles in BHK cells using Semliki Forest virus-derived RNA expression vectors. Proc. Natl. Acad. Sci. USA 93:1165811663.

20.Lieber, A., D.S. Steinwaerder, C.A. Carlson, and M.A. Kay. 1999. Integrating adenovirusadeno-associated virus hybrid vectors devoid of all viral genes. J. Virol. 73:9314-9324.

21.Lim, F., D. Hartley, P. Starr, P. Lang, S. Song, L. Yu, Y. Wang, and A.I. Geller. 1996. Generation of high-titer defective HSV-1 vectors using an IE 2 deletion mutant and quantitative study of expression in cultured cortical cells. BioTechniques 20:460-469.

22.Lim, F. and R. Neve. 1999. Generation of high-titer defective HSV-1 vectors, p. 4.13.1114.13.17. In G. Crooks (Ed.), Current Protocols in Neuroscience. Greene Publishing Associates and Wiley-Interscience, New York.

23.McCarthy, A.M., L. McMahan, and P.A. Schaffer. 1989. Herpes simplex virus type 1 ICP27 deletion mutants exhibit altered patterns of transcription and are DNA deficient. J. Virol. 63:18-27.

24.Morgenstern, J.P. and H. Land. 1990. Advanced mammalian gene transfer: high titer retroviral vectors with multiple drug selection markers and a complementary helper-free packaging cell line. Nucleic Acids Res. 18: 3587-3596.

25.Nieto, M.A., K. Patel, and D.G. Wilkinson. 1996. In situ hybridization analysis of chick embryos in whole mount and tissue sections. Methods Cell. Biol. 51:219-235.

26.Palombo, F., A. Monciotti, A. Recchia, R. Cortese, G. Ciliberto, and N. La Monica. 1998. Site-specific integration in mammalian cells mediated by a new hybrid baculovirusadeno-associated virus vector. J. Virol. 72:5025-5034.

27.Patience, C., Y. Takeuchi, F.-L. Cosset, and R.A. Weiss. 1998. Packaging of endogenous retroviral sequences in retroviral vectors produced by murine and human packaging cells. J. Virol. 72:2671-2676.

28. Ramsey, W.J., N.J. Caplen, Q. Li, J.N. Higginbotham, M. Shah, and R.M. Blaese. 1998. Adenovirus vectors as transcomplementing templates for the production of replication defective retroviral vectors. Biochem. Biophys. Res. Commun. 246:912-919.

29. Recchia, A., R.J. Parks, S. Lamartina, C. Toniatti, L. Pieroni, F. Palombo, G. Ciliberto, F.L. Graham et al. 1999. Site-specific integration mediated by a hybrid adenovirus/adeno-associated virus vector. Proc. Natl. Acad. Sci. USA 96:2615-2620.

30.Roemer, K., P.A. Johnson, and T. Friedmann. 1992. Recombination between a herpes simplex virus type 1 vector deleted for immediate early gene 3 and the infected cell genome. J. Gen. Virol. 73:1553-1558.

31.Saeki, Y., T. Ichikawa, A. Saeki, E.A. Chioc- ca, K. Tobler, M. Ackermann, X.O. Breakefield, and C. Fraefel. 1998. Herpes simplex virus type 1 DNA amplified as a bacterial artificial chromosome in Escherichia coli: rescue of replication-competent virus progeny and packaging of amplicon vectors. Hum. Gene Ther. 9:2787-2794.

32.Savard, N., F.L. Cosset, and A.L. Epstein. 1997. Defective herpes simplex virus type 1 vectors harboring gag, pol, and env genes can be used to rescue defective retrovirus vectors. J. Virol. 71:4111-4117.

33.Scadden, D.T., B. Fuller, and J.M. Cunningham. 1990. Human cells infected with retrovirus vectors acquire an endogenous murine provirus. J. Virol. 64:424-427.

34.Schwartzberg, P., J. Colicelli, and S.P. Goff. 1985. Recombination between a defective retrovirus and homologous sequences in host DNA: reversion by patch repair. J. Virol. 53:719-726.

35.Sena-Esteves, M., Y. Saeki, S.M. Camp, E.A. Chiocca, and X.O. Breakefield. 1999. Singlestep conversion of cells to retrovirus vector producers with herpes simplex virus-Epstein-Bar virus hybrid amplicons. J. Virol. 73:1042610439

36.Smith, I.L., M.A. Hardwicke, and R.M.Sandri-Goldin. 1992. Evidence that the herpes simplex virus immediate early protein ICP27 acts post-transcriptionally during infection to regulate gene expression. Virology 186:74-86.

37.Soneoka, Y., P.M. Cannon, E.E. Ramsdale, J.C. Griffiths, G. Romano, S.M. Kingsman, and A.J. Kingsman. 1995. A transient threeplasmid expression system for the production of high titer retroviral vectors. Nucleic Acids Res. 23:628-633.

38.Southern, E.M. 1975. Detection of specific sequences among DNA fragments separated by gel electrophoresis. J. Mol. Biol. 98:503-517.

39.Spaete, R.R. and N. Frenkel. 1982. The herpes simplex virus amplicon: a new eucaryotic defective-virus cloning-amplifying vector. Cell 30:295-304.

40.Starr, P.A., F. Lim, F.D. Grant, L. Trask, P. Lang, L. Yu, and A.I. Geller. 1996. Longterm persistence of defective $\mathrm{HSV}-1$ vectors in the rat brain is demonstrated by reactivation of vector gene expression. Gene Ther. 3:615-623.

41.Stavropoulos, T.A. and C.A. Strathdee. 1998. An enhanced packaging system for helper-dependent herpes simplex virus vectors. J. Virol. 72:7137-7143.

42.Steinwaerder, D.S., C.A. Carlson, and A. Lieber. 1999. Generation of adenovirus vectors devoid of all viral genes by recombination between inverted repeats. J. Virol. 73:9303-9313.

43.Stowe, N. and E. McMonagle. 1982. Propagation of foreign DNA sequences linked to a herpes simplex virus origin of replication, p. 199 204. In Y. Gluzman (Ed.), Eukaryotic Viral Vectors. CSH Laboratory Press, Cold Spring Harbor, NY.

44. Vazny, D.A., A. Kwong, and N. Frenkel. 1982. Site-specific cleavage/packaging of herpes simplex virus DNA and the selective maturation of nucleocapsids containing full-length viral DNA. Proc. Natl. Acad. Sci. USA 79:1423-1427.

45.Wahlfors, J.J., K.G. Xanthopoulos, and R.A. Morgan. 1997. Semliki Forest virus-mediated production of retroviral vector RNA in retroviral packaging cells. Hum. Gene Ther. 8:20312041.

46.Wang, S. and J.M. Vos. 1996. A hybrid herpesvirus infectious vector based on EpsteinBarr virus and herpes simplex virus type 1 for gene transfer into human cells in vitro and in vivo. J. Virol. 70:8422-8430.

47.Wang, X., G.R. Zhang, T. Yang, W. Zhang, and A.I. Geller. 2000. Fifty-one kilobase HSV1 plasmid vector can be packaged using a helper virus-free system and supports expression in the rat brain. BioTechniques 28:102107.

48. Wilson, C.A. and M.V. Eiden. 1991. Viral and cellular factors governing hamster cell infection by murine and gibbon ape leukemia viruses. J. Virol. 65:5975-5982.

49.Zheng, C., B.J. Baum, M.J. Iadarola, and B.C. O'Connell. 2000. Genomic integration and gene expression by a modified adenoviral vector. Nat. Biotechnol. 18:176-180.

Received 24 April 2000; accepted 25 January 2001.

Address correspondence to:

Dr. Filip Lim

Centro de Biología Molecular

Universidad Autónoma de Madrid

28049 Madrid, Spain

e-mail: flim@cbm.uam.es 